\title{
Multiple model updating using the finite element method over a polynomial algebra
}

\author{
C. Gouttebroze ${ }^{1}$, F. Louf ${ }^{1}$, L. Champaney ${ }^{1}$ \\ ${ }^{1}$ LMT-Cachan (ENS Cachan/CNRS/University Paris 6/UniverSud Paris) \\ 61 avenue du President Wilson, F-94230 Cachan, France
}

\begin{abstract}
Vibration control is an important issue when it comes to preserving the structural integrity of mechanical structures, particularly in the case of lightweight structures found aboard flying vehicles, such as printed circuit boards. In order for a simulation to be effective, a suitable numerical model of the structure must be used. Such a model can be obtained using a model updating method, such as that which is presented in this work, based on the modified constitutive relation error principle.

However, like many other model updating strategies, this method can be very expensive. When the structural parameters change over time, it would be too timeconsuming to perform another full calculation. In order to circumvent this problem, we introduce a new technique called the 'finite element method over a polynomial algebra', which involves modules defined over a ring of truncated polynomials in multiple variables.

In this paper, we illustrate the method with the updating and reduction of a model of a beam instrumented with piezoelectric sensors and actuators, which are all taken into account in the numerical model. This is a simple problem, yet it is representative of the updating of printed circuit boards.
\end{abstract}

\section{Introduction}

In the field of mechanics applied to the development of intelligent systems, the main areas of investigation are the control of the health or integrity of structures, the control of their shape, and active vibration damping. In this paper, we consider the case of lightweight structures, such as the printed circuit boards found aboard flying vehicles (planes, rockets,...). In the different flight configurations (liftoff, landing,...), these cards are subjected to low-frequency vibrations which can damage the components and electrical connections, causing them to age prematurely. The classical remedy, based on passive damping, works in only a limited range of frequencies and increases the weight significantly. A solution based on active damping allows the vibrations to be reduced in a wide frequency range while keeping the increase in weight moderate. Generally speaking, the active control process can be divided into three steps. First, the displacements of the structure are measured by sensors and a data processing system: this is the observation phase, in which the most commonly used sensors are piezoelectric components. Then comes the thinking phase, which consists in the elaboration of an answer. Finally, 
in the actuation phase, the resulting command is delivered to the structure. Again, the most commonly used actuators in this phase are piezoelectric components. The thinking phase requires an adequate model in order to deliver a satisfactory answer. In the case of lightweight structures, this model must take into account the presence of the piezoelectric components. One possible method to obtain such a model consists in using a model updating strategy.

A state-of-the-art review of finite element model updating methods can be
found in [1]. They all rely on stiffness, damping an mass matrices obtained
through changes in the physical parameters of the model. Their differences
come from the objective function they use. A first group of techniques try
to minimize input residuals like the Sensitivity-Based Element-by-Element
Updating Procedure [2] or the Equilibrium Gap Method [3]. The second group
is based on output residuals like the Modal Assurance Criterion [4] or the
Reciprocity Gap Method [5]. A third group relies on the residual over the
constitutive equations like the Constitutive Gap Method [6] or the Modified
Constitutive Relation Error (MCRE) [7], the one we use in this paper.
first developments of this method demonstrated the effectiveness of such a model in structural dynamics, then in forced vibrations with updated models obtained from eigenmodes. This approach, which uses the concept of Drucker error, provides an effective means of updating mass and stiffness matrices. Its governing principle consists in partitioning the equations of the problem into two groups, a reliable group and an uncertain group. Then, among the mechanical fields which satisfy the equations of the reliable group exactly, one seeks to minimize a norm containing the residuals of the uncertain group and the distance between the observed experimental data and the numerical calculation. Like many other popular updating techniques, it is an optimization-based method whose objective function is very computation-intensive.

The structural parameters of the structure can change over time due to ageing, damage, relative movements in the joints,... As this happens, a previous vibration control can become ineffective. In order to avoid going through another expensive model updating process, we propose to use a multivariate analysis approach. In this work, we introduce a new multivariate analysis technique called the Finite Element Method over a Polynomial Algebra (FEMPA). In this method, the coefficients of the vectors of the finite element basis are polynomials in multiple variables of interest.

First, we present the MCRE, the model updating method used here. Then, we introduce the FEMPA and describe its application to the one-time updating of a model of a structure representing a printed circuit board. Finally, we illustrate how the FEMPA can be used in order to update a structure rapidly when its parameters change over time. 


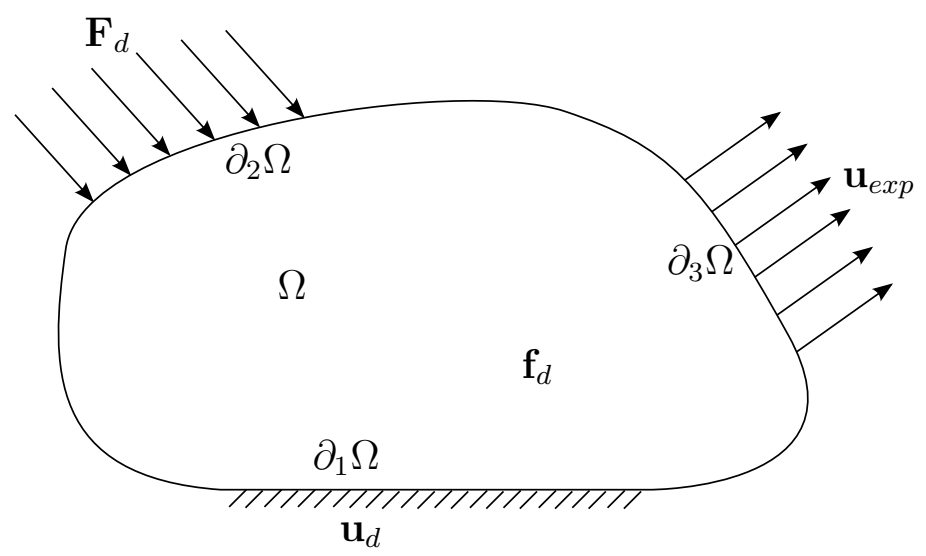

Figure 1. The domain being studied

\section{Presentation of the model updating method}

\subsection{The reference problem}

For the sake of simplicity, we present the model updating method using a viscoelastic structure as an example (see Figure 1). The application of the method to a viscoelastic piezoelectric structure was studied in [8]. The structure vibrates over a time interval $[0, T]$. It is subjected to body forces $\mathbf{f}_{d}$, prescribed displacements $\mathbf{u}_{d}$ over $\partial_{1} \Omega$ and distributed forces $\mathbf{F}_{d}$ over the boundary $\partial_{1} \Omega$.

The reference problem consists in finding a solution in terms of $s(\mathbf{x}, t)=$ $(\mathbf{u}(\mathbf{x}, t), \sigma(\mathbf{x}, t), \boldsymbol{\Gamma}(\mathbf{x}, t))$ (the displacement, stress and acceleration quantities respectively), with $\mathbf{x} \in \Omega, t \in[O, T]$, which satisfies the kinematic constraints:

$$
\mathbf{u}_{\mid \partial_{1} \Omega}=\mathbf{u}_{d}
$$

the equilibrium equations:

$$
\begin{gathered}
\nabla \sigma+\mathbf{f}_{d}=\boldsymbol{\Gamma}, \\
\sigma \mathbf{n}_{\mid \partial_{2} \Omega}=\mathbf{F}_{d},
\end{gathered}
$$

and the constitutive equations:

$$
\begin{aligned}
& \sigma=\mathcal{K}_{\mathbb{E}}(\mathbf{u})+\mathcal{D}_{\mathbb{E}(\dot{\mathbf{u}}),} \\
& \Gamma=\rho \ddot{\mathbf{u}} .
\end{aligned}
$$

$\mathcal{K}$ denotes Hooke's tensor, $\mathcal{D}$ the damping tensor and $\rho$ the density. These quantities can be defined by a set of $n_{p}$ structural parameters. During an experimental test, one measures a displacement $\mathbf{u}_{\text {exp }}$ over $\partial_{3} \Omega$. In order to agree with the experiments, the solution $s(\mathbf{x}, t)$ must satisfy:

$$
\mathbf{u}_{\mid \partial_{3} \Omega}=\mathbf{u}_{e x p}
$$




\subsection{The Modified Constitutive Relation Error approach}

The MCRE concept, like the CRE concept, is based on a partitioning of the equations into reliable equations and less reliable equations. The difference comes from the presence of equation (6), which introduces a distance between the numerical model and the experimental data. For a given set of structural parameters, a displacement field $\mathbf{u}_{a d}$ is said to be kinematically admissible if it satisfies (1). Stress and acceleration fields $\sigma_{a d}, \boldsymbol{\Gamma}_{a d}$ are said to be dynamically admissible if they satisfy (2) and (3). The MCRE approach consists in finding an admissible solution $s_{a d}=\left(\mathbf{u}_{a d}, \sigma_{a d}, \boldsymbol{\Gamma}_{a d}\right)$ which minimizes the residuals of (4), (5) and (6):

$$
\begin{aligned}
e^{2}(s)= & \gamma \int_{t=0}^{T}\|\mathcal{K} \mathbb{E}(\mathbf{u})+\mathcal{D} \mathbb{E}(\dot{\mathbf{u}})-\sigma\|^{2} \mathrm{~d} t \\
& +(1-\gamma) \int_{t=0}^{T}\|\rho \ddot{\mathbf{u}}-\boldsymbol{\Gamma}\|^{2} \mathrm{~d} t \\
& +\frac{r}{1-r} \int_{t=0}^{T}\left\|\mathbf{u}-\mathbf{u}_{\text {exp }}\right\|^{2} \mathrm{~d} t
\end{aligned}
$$

where $\gamma \in[0,1]$ and $r \in[0,1[$ are weighting parameters. Parameter $r$ represents one's degree of confidence in the experimental data. The term $\frac{r}{1-r} \in[0, \infty[$ is used to apply the desired weight to the experimental part of the error, even if its initial magnitude is very different from that of the other errors. Different versions of the norms involved in equation (7) can be found in [9]. The discrete form of the norms we are using in this paper will be presented in equation (15).

In the case of a forced vibration problem at an angular frequency $\omega$, the calculation of the objective function over a duration $T^{\prime}$ which is a multiple of the period leads to:

$e_{\omega}^{2}(s)=\frac{\gamma}{2}\left\|\left(\mathcal{K}+T^{\prime} \omega^{2} \mathcal{D}\right) \mathbb{E}(\mathbf{u})-\sigma\right\|^{2}+\frac{1-\gamma}{2}\left\|\rho \omega^{2} \mathbf{u}-\boldsymbol{\Gamma}\right\|^{2}+\frac{r}{1-r}\left\|\mathbf{u}-\mathbf{u}_{\text {exp }}\right\|^{2}$.

\subsection{Discretized form of the modified error}

For numerical convenience, the problem can be described in terms of displacements by introducing fields $\underline{v}_{a d}$ and $\underline{w}_{a d}$ such that the associated stress and acceleration fields are dynamically admissible:

$$
\begin{aligned}
& \sigma=\mathcal{K} \mathbb{E}\left(\underline{v}_{a d}\right)+\mathcal{D} \mathbb{E}\left(\dot{\mathbf{v}}_{a d}\right), \\
& \Gamma=\rho \ddot{\mathbf{w}}_{a d} .
\end{aligned}
$$

Now, let us move on and discretize the problem. We introduce a finite element basis $\{\bowtie\}=\left\{\boldsymbol{\phi}_{i}\right\}_{i=1, \ldots, n_{\text {dof }}}$ such that the displacement fields are approximated in the form:

$$
\mathbf{u}_{a d}=\sum_{i=1}^{n_{\text {dof }}} u_{i} \boldsymbol{\phi}_{i}, \quad \underline{v}_{a d}=\sum_{i=1}^{n_{\text {dof }}} v_{i} \boldsymbol{\phi}_{i}, \quad \underline{w}_{a d}=\sum_{i=1}^{n_{\text {dof }}} w_{i} \boldsymbol{\phi}_{i}
$$


where $u_{i}, v_{i}$ and $w_{i}$ denote the $i^{t h}$ coefficients of the vectors of the nodal displacements $\{U\},\{V\}$ and $\{W\}$ respectively. We build the discretized stiffness matrix $[\mathbf{K}]$, damping matrix $[\mathbf{D}]$ and mass matrix $[\mathbf{M}]$ such that their $i^{t h}$-line and $j^{t h}$-column coefficients are:

$$
\begin{aligned}
{\left[\mathbf{K}_{i j}\right] } & =\int_{\Omega} \operatorname{Tr}\left[\mathcal{K} \mathbb{E}\left(\phi_{i}\right) \mathbb{E}\left(\boldsymbol{\phi}_{j}\right)\right] \mathrm{d} V, \\
{\left[\mathbf{D}_{i j}\right] } & =\int_{\Omega} \operatorname{Tr}\left[\mathcal{D} \mathbb{E}\left(\boldsymbol{\phi}_{i}\right) \mathbb{E}\left(\boldsymbol{\phi}_{j}\right)\right] \mathrm{d} V, \\
{\left[\mathbf{M}_{i j}\right] } & =\int_{\Omega} \rho \boldsymbol{\phi}_{i} \cdot \boldsymbol{\phi}_{j} \mathrm{~d} V .
\end{aligned}
$$

With these notations, the discrete form of $e_{\omega}^{2}(s)$ is given by:

$$
\begin{aligned}
e_{\omega}^{2}(\{U\},\{V\},\{W\})= & \frac{\gamma}{2}\{U-V\}^{\star}\left([\mathbf{K}]+T^{\prime} \omega^{2}[\mathbf{D}]\right)\{U-V\} \\
& +\frac{1-\gamma}{2} \omega^{2}\{U-W\}^{\star}[\mathbf{M}]\{U-W\} \\
& +\frac{r}{1-r}\left\{\Pi_{3} U-U_{\text {exp }}\right\}^{\star}[\mathbf{G}]\left\{\Pi_{3} U-U_{\text {exp }}\right\},
\end{aligned}
$$

where $^{*}$ is the conjugate transpose operator, and $\Pi_{3}$ a projection operator onto $\partial_{3} \Omega$. Matrix $[\mathbf{G}]$ quantifies the error in the measurements and can be expressed as:

$$
[\mathbf{G}]=\frac{\gamma}{2} \Pi_{3}\left([\mathbf{K}]+T^{\prime} \omega^{2}[\mathbf{D}]\right) \Pi_{3}^{\star}+\frac{1-\gamma}{2} \omega^{2} \Pi_{3}[\mathbf{M}] \Pi_{3}^{\star} .
$$

In addition, the solution $(\{U\},\{V\},\{W\})$ must be admissible, i.e. it must satisfy:

$$
\begin{aligned}
& \Pi_{1}\{U\}=\left\{U_{d}\right\}, \\
& ([\mathbf{K}]+\mathrm{i} \omega[\mathbf{D}])\{V\}-\omega^{2}[\mathbf{M}]\{W\}=\{F\},
\end{aligned}
$$

where $\Pi_{1}$ is a projection operator onto $\partial_{1} \Omega$ and $\{F\}$ is the excitation force vector. The expression of $e_{\omega}^{2}(s)$ under the admissibility constraints is obtained through the introduction of Lagrange multipliers. Then, for a given a set of the structural parameters, one can calculate $\{U\},\{V\}$ and $\{W\}$. During these calculations, which are not described in detail here, the Lagrange multiplier corresponding to equation (18) is eliminated and the change of variables from $(\{U\},\{V\},\{W\})$ to $(\{U\},\{U-V\},\{U-$ $W\}$ ) comes naturally. We end up with the resolution of a system of linear equations $[A]\{X\}=\{B\}$ with:

$$
\begin{aligned}
& A=\left[\begin{array}{cccc}
\frac{\gamma}{2}\left([\mathbf{K}]+T^{\prime} \omega^{2}[\mathbf{D}]\right) & \frac{1-\gamma}{2} \omega^{2}[\mathbf{M}] & \frac{r}{1-r}[\mathbf{G}] & 0 \\
\frac{\gamma}{2}\left([\mathbf{K}]+T^{\prime} \omega^{2}[\mathbf{D}]\right) & \frac{1-\gamma}{2}([\mathbf{K}]+\mathrm{i} \omega[\mathbf{D}]) & 0 & 0 \\
-([\mathbf{K}]+\mathrm{i} \omega[\mathbf{D}]) & \omega^{2}[\mathbf{M}] & {[\mathbf{K}]+\mathrm{i} \omega[\mathbf{D}]-\omega^{2}[\mathbf{M}]} & \Pi_{1}^{T} \\
0 & 0 & \Pi_{1} & 0
\end{array}\right] \\
& X=\left\{\begin{array}{c}
\{U-V\} \\
\{U-W\} \\
\{U\} \\
\{\lambda\}
\end{array}\right\}, \quad B=\left\{\begin{array}{c}
\frac{r}{1-r}[\mathbf{G}]\left\{U_{\text {exp }}\right\} \\
0 \\
\{F\} \\
\left\{U_{d}\right\}
\end{array}\right\}
\end{aligned}
$$

Once the fields $\{U\},\{V\}$ and $\{W\}$ have been determined, the value of the objective function is calculated using equation (15). 


\section{The finite element method over a polynomial algebra}

\subsection{The two families of model reduction techniques}

Let us consider a problem discretized spatially into $n_{\text {dof }}$ degrees of freedoms in a frequency range discretized with $n_{\omega}$ points. For any set of the structural parameters which define the stiffness, damping and mass matrices, the calculation of the objective function requires the resolution of $n_{\omega}$ linear systems of size $3 n_{\text {dof }}$ (see equation (20)). If $n_{\text {dof }}$ and/or $n_{\omega}$ are large, the objective function

$$
e^{2}\left(s_{a d}\right)=\sum_{\omega=\omega_{1}}^{\omega_{n_{\omega}}} e_{\omega}^{2}\left(s_{a d}\right)
$$

must be calculated approximately. There are two families of techniques, leading to different kinds of approximations.

The first family, based on a mechanical approach to the problem, consists in reducing $n_{\text {dof }}$, which can be done by reducing the structure to its eigenmodes in a frequency range of interest [4]. Alternatively, one can use a condensation method. The second family, based on a mathematical approach to the problem, consists in replacing the objective function by a simpler function. The most natural way to do that is to compute a Taylor series expansion of the objective function in the structural parameters, which can be done by three differents approaches.

The easiest maner to perform it is to have a sampling approach $[10,11]$. It is based on the evalution of the objective function for $n_{\text {samp }}$ sets of the structural parameters. Thus is requires the resolution of $n_{\text {samp }} n_{\omega}$ linear systems of size $3 n_{\text {dof }}$. It is easy to implement but the choice of the sampling points can be a sentitive issue for the quality of the Taylor series expansion. This problem is avoided by the techniques based on the perturbation theory, like the perturbation finite element method $[12,13]$. They introduce the parametric variabilities into the numerical model under variational form. It leads to the resolution of $n_{\text {omega }}$ linear systems of size $3 n_{\text {dof }} n_{\text {samp }}^{\prime}$, where $n_{\text {samp }}^{\prime}$ is the dimension of the Taylor series expansion. The main drawback is the needed of heavy software development in order to change the basis where the solution is seeked.

We introduce here a new method to obtain the Taylor series expansion of the objective function, called the Finite Element Method over a Polynomial Algebra (FEMPA), that keeps the dependence over the parameters without changing the finite element basis. It is done by changing the ring over which the finite element method is build, using a multivariate polynomial ring instead of the real field or the complex field. It leads to the resolution of $n_{\text {omega }}$ linear systems of size $3 n_{\text {dof }}$ which coefficients are polynomials. 


\subsection{The polynomial algebra}

Let $\mathcal{P}\left(n_{d}, n_{X}, \mathbb{K}\right)$ denote the set of the polynomials in $n_{X}$ variables with maximum degree $n_{d}$ and coefficients in $\mathbb{K}$. In the rest of the paper, for the sake of simplicity, this set will be denoted $\mathcal{P}$ when there is no ambiguity. The $n_{X}$ variables will be denoted $\mathbf{X}=\left(X_{1}, \ldots, X_{n_{X}}\right)$. A polynomial $p \in \mathcal{P}$ can be expressed as:

$$
p=\sum_{\mathbf{i} \leqslant n_{d}} p_{\mathbf{i}} \mathbf{X}^{\mathbf{i}}=\sum_{i_{1}+\ldots+i_{n_{X}} \leqslant n_{d}} p_{i_{1}, \ldots, i_{n_{X}}} X_{1}^{i_{1}} \ldots X_{n_{X}}^{i_{n_{X}}} .
$$

For $k \in \mathbb{K}$ and $(p, q) \in \mathcal{P}^{2}$,

$$
p=\sum_{\mathbf{i} \leqslant n_{d}} p_{\mathbf{i}} \mathbf{X}^{\mathbf{i}}, q=\sum_{\mathbf{i} \leqslant n_{d}} q_{\mathbf{i}} \mathbf{X}^{\mathbf{i}},
$$

let + denote the internal operation

$$
p+q=\sum_{\mathbf{i} \leqslant n_{d}}\left(p_{\mathbf{i}}+q_{\mathbf{i}}\right) \mathbf{X}^{\mathbf{i}},
$$

let . denote the external operation

$$
k \cdot p=\sum_{\mathbf{i} \leqslant n_{d}} k p_{\mathbf{i}} \mathbf{X}^{\mathbf{i}}
$$

and let $\times$ denote the internal operation

$$
p \times q=\sum_{\mathbf{i} \leqslant n_{d}}\left(\sum_{(\boldsymbol{\alpha}, \boldsymbol{\beta}) \in \Sigma_{\mathbf{i}}} p_{\boldsymbol{\alpha}} q_{\boldsymbol{\beta}}\right) \mathbf{X}^{\mathbf{i}},
$$

where $\Sigma_{\mathbf{i}}$ is the set:

$$
\Sigma_{\mathbf{i}}=\left\{(\boldsymbol{\alpha}, \boldsymbol{\beta}) \mid \forall s \in\left[1, \ldots, n_{X}\right], \alpha_{s}+\beta_{s}=i_{s}\right\} .
$$

With these definitions, it is very easy to prove that $(\mathcal{P},+, ., \times)$ has the required properties to be an algebra over the field $\mathbb{K}$ and that $(\mathcal{P},+, \times)$ as the required properties to be a ring. It is not a field because an element has an inverse for operation $\times$ if, and only if, its constant term is nonzero. Let $\mathcal{P} \div$ denote the subset of $\mathcal{P}$ whose elements have an inverse for operation $\times$. We will show that $\mathcal{P}^{\div} \neq \mathcal{P}-\left\{0_{\mathcal{P}}\right\}$, where $0_{\mathcal{P}}$ is the neutral element of operation + .

For a given $p \in \mathcal{P}$, denoting $1_{\mathcal{P}}$ is the neutral element of operation $\times$, one can find $q \in \mathcal{P}$ such that:

$$
p \times q=\left(\sum_{\mathbf{i} \leqslant n_{d}} p_{\mathbf{i}} \mathbf{X}^{\mathbf{i}}\right) \times\left(\sum_{\mathbf{i} \leqslant n_{d}} q_{\mathbf{i}} \mathbf{X}^{\mathbf{i}}\right)=1_{\mathcal{P}}
$$

when

$$
q_{\mathbf{i}}= \begin{cases}\frac{1}{p_{(0, \ldots, 0)}} & \text { for } \mathbf{i}=(0, \ldots, 0) \\ \frac{-1}{p_{(0, \ldots, 0)}} \sum_{(\boldsymbol{\alpha}, \boldsymbol{\beta}) \in \Sigma_{\mathbf{i}}^{\prime}} p_{\boldsymbol{\alpha}} q_{\boldsymbol{\beta}} & \text { for } \mathbf{i} \neq(0, \ldots, 0)\end{cases}
$$




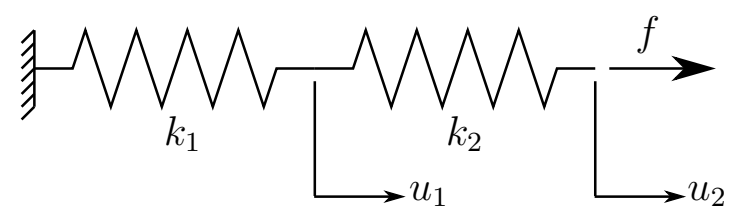

Figure 2. Two springs

where $\Sigma_{\mathbf{i}}^{\prime}$ is the set:

$$
\Sigma_{\mathbf{i}}=\left\{(\boldsymbol{\alpha}, \boldsymbol{\beta}) \mid(\boldsymbol{\alpha}, \boldsymbol{\beta}) \in \Sigma_{\mathbf{i}} \text { and } \boldsymbol{\alpha} \neq(0, \ldots, 0)\right\} .
$$

In other words, such a polynomial $q$ exists if, and only if, $p_{(0, \ldots, 0)} \neq 0$. In such a case, we will denote $q=1_{\mathcal{P}} \div p$.

\subsection{An example of application}

Let us consider a series of two springs (see Figure 2) whose stiffnesses $k_{1}$ and $k_{2}$ vary around values $k_{1}^{(0)}$ and $k_{2}^{(0)}$ respectivly. In order to apply the FEMPA, we express the stiffnesses as :

$$
k_{1}(\mathbf{X})=k_{1}^{(0)}\left(1+X_{1}\right), \quad k_{2}(\mathbf{X})=k_{2}^{(0)}\left(1+X_{2}\right) .
$$

If additional information is available concerning the variation of these stiffnesses, for example following two functions $g_{1}$ and $g_{2}$, one can use the Taylor series expansions of functions $g_{1}$ and $g_{2}$ around $k_{1}^{(0)}$ and $k_{2}^{(0)}$.

Let us now apply a force $f$ to the second spring. The problem can be described using the equations:

$$
\left\{\begin{array}{rl}
k_{1} u_{1}-k_{2}\left(u_{2}-u_{1}\right) & =0 \\
k_{2}\left(u_{2}-u_{1}\right) & =f
\end{array} .\right.
$$

This problem can be rewritten in matrix form. In the framework of the FEMPA, this leads to matrices and vectors whose coefficients belong to $\mathcal{P}$ :

$$
\left[\begin{array}{cc}
k_{1}^{(0)}\left(1+X_{1}\right)+k_{2}^{(0)}\left(1+X_{2}\right) & -k_{2}^{(0)}\left(1+X_{2}\right) \\
-k_{2}^{(0)}\left(1+X_{2}\right) & k_{2}^{(0)}\left(1+X_{2}\right)
\end{array}\right]\left\{\begin{array}{l}
u_{1}\left(X_{1}, X_{2}\right) \\
u_{2}\left(X_{1}, X_{2}\right)
\end{array}\right\}=\left\{\begin{array}{l}
0 \\
f
\end{array}\right\} .
$$

The analytic solution of this system is:

$$
\left\{\begin{array}{l}
u_{1}(\underline{\theta})=f \frac{1}{k_{1}} \\
u_{2}(\underline{\theta})=f \frac{k_{1}+k_{2}}{k_{1} \cdot k_{2}}
\end{array},\right.
$$

which can be rewritten, using the FEMPA notations, in the form:

$$
\left\{\begin{array}{l}
u_{1}(\mathbf{X})=f \div\left(k_{1}^{(0)}\left(1+X_{1}\right)\right) \\
u_{2}(\mathbf{X})=f \times\left(k_{1}^{(0)}\left(1+X_{1}\right)+k_{2}^{(0)}\left(1+X_{2}\right)\right) \div\left(k_{1}^{(0)}\left(1+X_{1}\right) \times k_{2}^{(0)}\left(1+X_{2}\right)\right)
\end{array} .\right.
$$




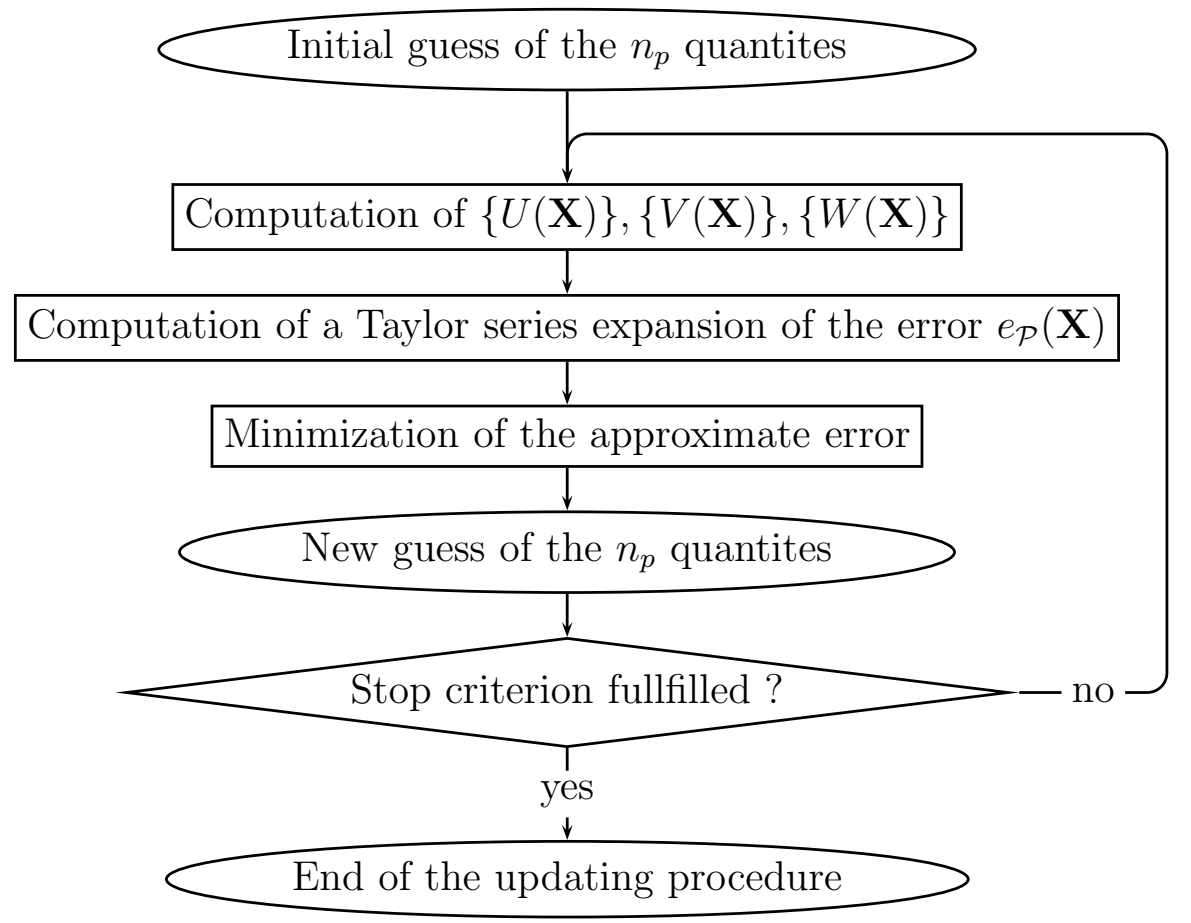

Figure 3. Algorithme de recalage suivant une stratgie algbrique

During the resolution of the linear system (33) using a specific algorithm, the elementary operations leading to the solution (35) are carried out with the definitions given in equations (23), (26) and (29). When the degree $n_{d}$ is equal to 2 , we obtain the solution:

$$
\left\{\begin{array}{l}
u_{1}\left(X_{1}, X_{2}\right)=\frac{f}{k_{1}^{(0)}}-\frac{f}{k_{1}^{(0)}} X_{1}+\frac{f}{k_{1}^{(0)}} X_{1}^{2} \\
u_{2}\left(X_{1}, X_{2}\right)=\frac{f}{k_{1}^{(0)}}+\frac{f}{k_{2}^{(0)}}-\frac{f}{k_{1}^{(0)}} X_{1}+\frac{f}{k_{1}^{(0)}} X_{1}^{2}-\frac{f}{k_{2}^{(0)}} X_{2}+\frac{f}{k_{2}^{(0)}} X_{2}^{2}
\end{array}\right.
$$

If one needs to calculate a quantity of interest using the vectors of the nodal displacements $\left[u_{1}, u_{2}\right]^{T}$, one can also use the operations defined in Subsection 3.2. 


\section{Application of the FEMPA to the one-time updating of a model}

\subsection{The strategy}

In order to apply the FEMPA to the one-time updating of a model, we write the $n_{p}$ structural parameters under polynomial form as in equation (31). Thus, the $i$-th quantity we want to update $z_{i}$ is written:

$$
z_{i}(\mathbf{X})=z_{i}^{(0)}\left(1+X_{i}\right)
$$

where $z_{i}^{(0)}$ is an initial guess of this quantity. In order to compute a polynomial form of the displacement $\{U(\mathbf{X})\},\{V(\mathbf{X})\},\{W(\mathbf{X})\}$ we solve the linear system (20) whose coefficients lie now in $\mathcal{P}\left(n_{d}, n_{p}, \mathbb{R}\right)$, using the definitions given in equations (23), (26) and (29). We also use them and equation (15) to compute a first Taylor series expansion of the error :

$$
e \approx e_{\mathcal{P}}^{(0)}(\mathbf{X})=e_{\mathcal{P}}^{(0)}\left(X_{1}, \ldots, X_{n_{p}}\right)
$$

Then, we try to find a local minimum which is close to $(0, \ldots, 0)$, denoted $\mathbf{a}^{(1)}=\left(a_{1}^{(1)}, \ldots, a_{n_{p}}^{(1)}\right)$ when it exists. The minimization algorithm choice is given to the reader, but a variable-step BFGS algorithm is recommended, with the identity matrix as the initial guess for the Hessian matrix. Once the minimum is found, we have a new guess of the quantity we want to update, the $i$-th being:

$$
z_{i}^{(1)}=z_{i}^{(0)}\left(1+a_{i}^{(1)}\right)
$$

which can be rewritten under polynomial form

$$
z_{i}(\mathbf{X})=z_{i}^{(1)}\left(1+X_{i}\right)
$$

in order to repeat all the steps described above until convergence as described in figure 3.

\subsection{Presentation of the example}

Let us consider an aluminum isotropic beam instrumented with one piezoelectric sensor and one piezoelectric actuator. Both piezoelectric components are assumed to be transverse isotropic. All the dimensions are shown in Figure 4. The material properties are given in Table 1 for the beam and in Table 2 for the piezoelectric components.

With this structure, we tested our model updating method by modifying the Young's modulus and the structural damping of the beam. The experimental measurements were simulated by perturbing the structural parameters by:

$$
E^{(e x p)}=0.7 E, \quad D^{(\exp )}=1.4 D .
$$

We obtained a Frequency Response Function (FRF). In order to limit the number of frequencies used to calculate the error (see equation (21)), we retained only the measured frequencies with high FRF amplitude: this is justified by the fact that in actual experiments it is at these frequencies that there is the least measured noise. In our example, the 4 first frequencies were $6,33,88$ and $178 \mathrm{~Hz}$. 


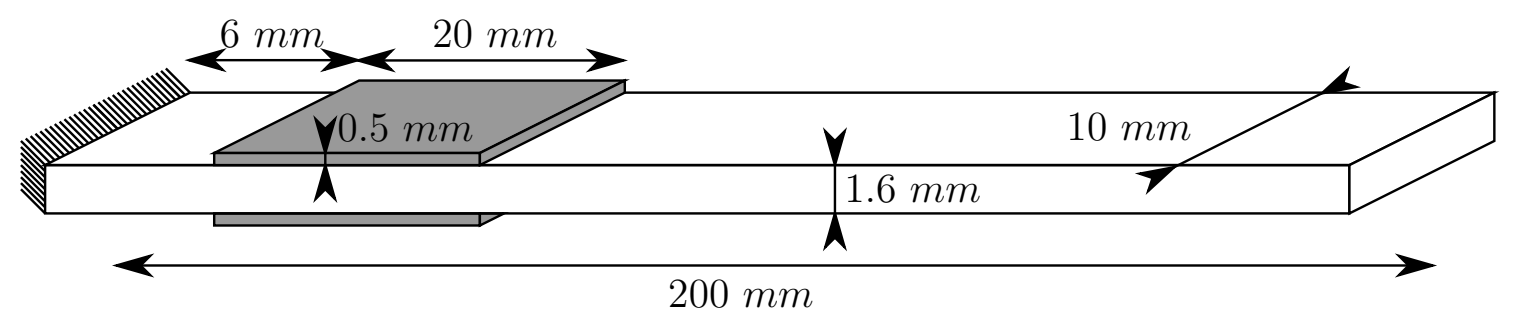

Figure 4. The instrumented beam

Table 1. Material properties of the beam

\begin{aligned} & \hline Parameter Value \\ & \hline Young's modulus $E=70 \mathrm{GPa} \\ &$ Shear modulus $26 \mathrm{GPa} \\ &$ Density $2,700 \mathrm{~kg} \cdot \mathrm{m}^{-3} \\ &$ Structural damping $D=0.7 \mathrm{MPa} . \mathrm{s} \\ &$ Structural shear damping $0.26 \mathrm{MPa.s} \\ &$\hline\end{aligned}

Table 2. Material properties of the piezoelectric components

\begin{tabular}{rl}
\hline Parameter & Value \\
\hline Young's modulus in Direction $\underline{e_{1}}$ & $127 \mathrm{GPa}$ \\
Shear modulus in Directions $\underline{e_{1}}, \underline{e_{3}}$ & $22.9 \mathrm{GPa}$ \\
Piezoelectric constant in Directions $\underline{\underline{e_{1}}}, \underline{\underline{e_{3}}}$ & $-6.62 \mathrm{~kg} \cdot \mathrm{m}^{-2}$ \\
Dielectric constant in Direction $\underline{\underline{e_{1}}}$ & $127.10^{-11} \mathrm{~F} . \mathrm{m}^{-1}$ \\
Density & $7,500 \mathrm{~kg} . \mathrm{m}^{-3}$ \\
Structural damping in Direction $\underline{\underline{e_{1}}}$ & $0.5 \mathrm{MPa} . \mathrm{s}$ \\
Shear damping in Directions $\underline{\underline{e_{1}}}, \underline{\underline{e_{3}}}$ & $0.5 \mathrm{MPa} . \mathrm{s}$ \\
\hline
\end{tabular}

In addition, we chose $r=\gamma=0.5$. The calculations of the successive minima of the error (see equations 38) was performed with a variable-step BFGS algorithm, with the identity matrix as the initial guess for the Hessian matrix.

\subsection{Results of the model updating}

The initial guess of the structural parameters was $E^{(0)}=E$ and $D^{(0)}=D$. Figure 5 shows the exact objective function (whose minimum is $\left(E^{(e x p)}, D^{(e x p)}\right)$ ) and its successive approximations with polynomials of degree 4 . Table 3 shows that the minimum was reached after three iterations. 


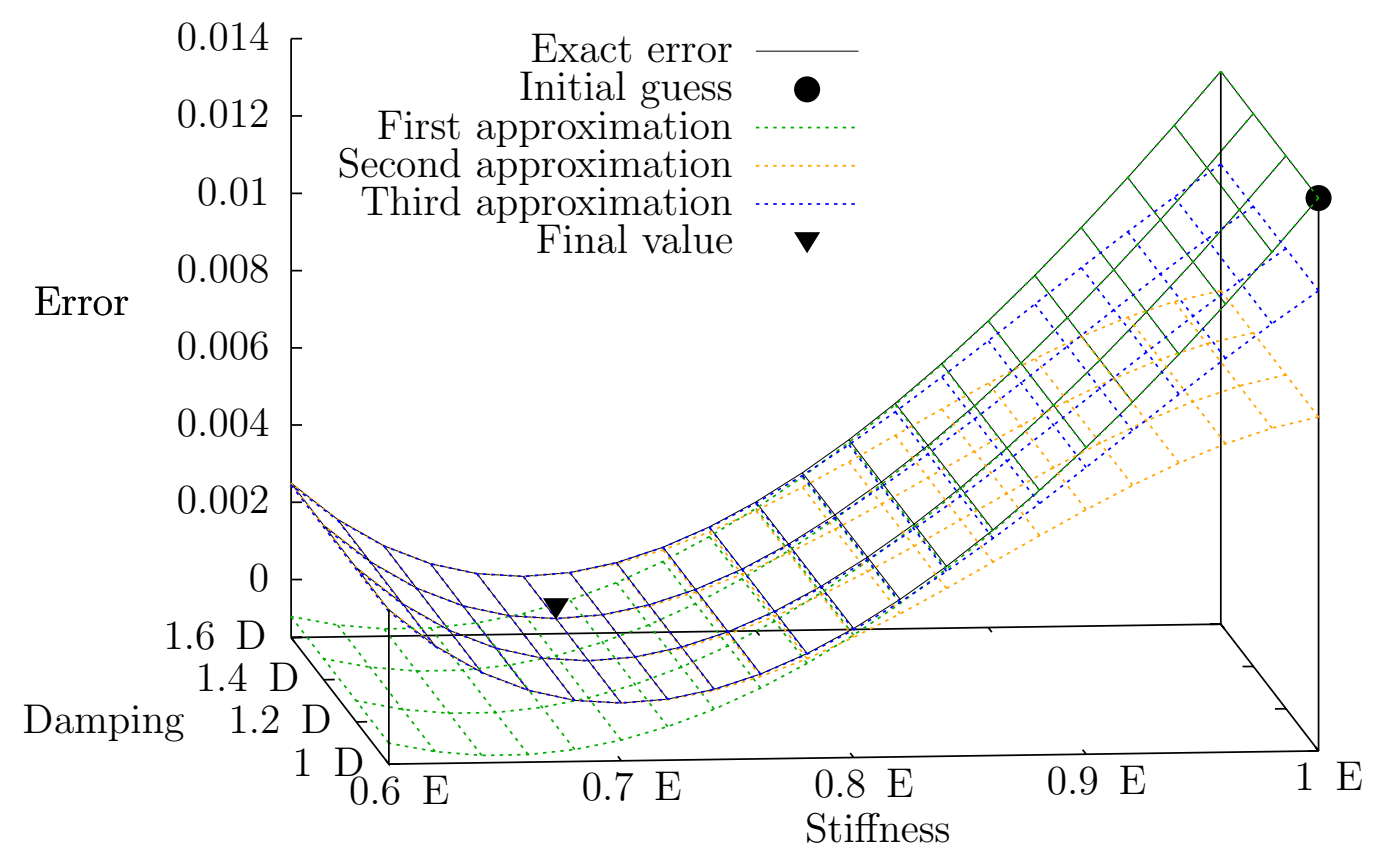

Figure 5. The objective function and its approximations

Table 3. The minima of the successive approximations

\begin{tabular}{ccc}
\hline$i$ & $\left(a_{1}^{(i)}, a_{2}^{(i)}\right)$ & $\left(E^{(i)}, D^{(i)}\right)$ \\
\hline 1 & $(-0.356099,0.587651)$ & $(0.643901 E, 1.58765 D)$ \\
2 & $(0.0868269,-0.118969)$ & $(0.699809 E, 1.39877 D)$ \\
3 & $(0.00027282,0.00087909)$ & $(0.7 E, 1.4 D)$ \\
\hline
\end{tabular}

Each calculation of an approximation of $e^{2}(s)$ took 31 seconds on a $2 \mathrm{GHz}$ dualcore processor with $512 \mathrm{Mb}$ RAM, using a code written in $\mathrm{C}++$. The total time of 93 seconds was 2.5 times less than with the usual scalar field method, which required 232 seconds to obtain results of similar quality, i.e. an error in the final guess of the parameters of less than $10^{-6}$. 
Table 4. Computation times in relation to polynomial degree

\begin{tabular}{cccc}
\hline $\begin{array}{c}\text { Polynomial } \\
\text { degree }\end{array}$ & $\begin{array}{c}\text { Time for one } \\
\text { approximation }\end{array}$ & $\begin{array}{c}\text { Number of } \\
\text { approximations }\end{array}$ & $\begin{array}{c}\text { Total } \\
\text { time }\end{array}$ \\
\hline 2 & $15 s$ & $\infty$ & $\infty$ \\
3 & $21 s$ & 5 & $105 s$ \\
4 & $31 s$ & 3 & $93 s$ \\
5 & $46 s$ & 3 & $138 s$ \\
6 & $73 s$ & 2 & $146 s$ \\
\hline
\end{tabular}

\subsection{The influence of the degree of the polynomials}

The degree nd of the polynomials has an influence on both the computation time and the quality of the approximation. As shown in Table 4, on the one hand, the lower the degree, the better the computation time; on the other hand, increasing the degree improves the quality of the approximation and, therefore, decreases the number of iterations needed to achieve convergence. For our problem, for which we sought an error in the final guess of the parameters of less than $10^{-6}$ for each polynomial degree, $n d=4$ was the best choice.

In some cases which are not addressed here, it may be useful to know how the computation time varies with the number of variables $n_{X}$. This variation is the same as the variation with the degree nd because they have symmetrical roles in the expression of the dimension of the algebra $\left(\mathcal{P}\left(n_{d}, n_{X}, \mathbb{K}\right),+, ., \times\right)$, which is $\frac{\left(n_{d}+n_{X}\right) !}{n_{d} ! n_{X} !}$.

\section{Application of the FEMPA to multiple model updating}

\subsection{General principle}

In order to update a structure whose behavior changes due to ageing effects, we proceed in two steps.

The first step consists in constructing an objective function which represents the evolution of the structure's behavior. In addition to expressing how the error varies with the structural parameters, we are interested in its dependence on the experimental data $\underline{u}_{e x p}$. In order to express this dependence, we rely on the measurments before ageing.

The second step consists in updating the model for each ageing stage using its specific measured FRF. In order to do that, we take the restriction of the objective function to the experimental data of the ageing stage we are interested in. Thus, we get a function whose only variables are the structural parameters and whose minimization cost is very small. 


\subsection{Construction of the objective function}

During $n_{\omega}$ tests at several frequencies, the real and imaginary parts $\underline{s}^{(0)}=$ $\left(s_{1}^{(0)}, \ldots, s_{2 n_{c} n_{\omega}}^{(0)}\right)$ of the measurements are collected and expressed in polynomial form:

$$
s_{i}^{(0)}(\underline{X})=s_{i}^{(0)}\left(1+X_{n_{p}+i}\right),
$$

for $i=1, \ldots, 2 n_{c} n_{\omega}$. (The shifted indices are due to the fact that the $n_{p}$ unknowns are retained for the quantities we want to update.) In order to get a Taylor series expansion of the error $e_{\mathcal{P}}^{(0)}\left(X_{1}, \ldots, X_{n_{p}+2 n_{c} n_{\omega}}\right)$, we solve the linear system (20) with coefficients in $\mathcal{P}\left(n_{d}, n_{p}+2 n_{c} n_{\omega}, \mathbb{C}\right)$, then use equation (15).

\subsection{Use of the objective function}

For the $k$-th ageing stage of the structure, a test leads to $2 n_{s} n_{\omega}$ measurements $s_{1}^{(k)}, \ldots, s_{2 n_{c} n_{\omega}}^{(k)}$ which are linked to the initial measurements through a set of parameters $\underline{\eta}^{(k)}=\left\{\eta_{i} \mid i=1, \ldots, 2 n_{c} n_{\omega}\right\}$ such that:

$$
s_{i}^{(k)}=s_{i}^{(0)}\left(1+\eta_{i}^{(k)}\right),
$$

for $i=1, \ldots, 2 n_{c} n_{\omega}$. This calculation cannot be carried out if $s_{i}^{0}=0$, which occurs when the sensor is on a node of the structure. In this case, it is necessary to move the sensor or to change the frequency of the experimental test. If neither is possible, the data from the particular sensor must be omitted in the calculation of the multi-structure error.

Then, we proceed with the calculation of the restriction of that error to the $k$-th ageing stage:

$$
e_{\mathcal{P}}^{(k)}\left(X_{1}, \ldots, X_{n_{p}}\right)=e_{\mathcal{P}}^{(0)}\left(X_{1}, \ldots, X_{n_{p}}, \eta_{1}^{(k)}, \ldots, \eta_{2 n_{c} n_{\omega}}^{(k)}\right)
$$

We seek the local minimum of $\varepsilon_{I_{\omega}}^{(k)}$ which is closest to $(0, \ldots, 0)$ and denote $\underline{\theta}^{(k)}$ when it exists. This enables us to find the values $\underline{z}^{(k)}$ of the quantities of the $k$-th ageing stage:

$$
\underline{z}_{i}^{(k)}=\underline{z}_{i}^{(0)}\left(1+\theta_{i}^{(k)}\right),
$$

for $i=1, \ldots, n_{p}$.

\subsection{Results}

We took the same example as in the previous section. The computation of the Taylor series expansion of the error $e_{\mathcal{P}}^{(0)}\left(X_{1}, \ldots, X_{n_{p}+2 n_{c} n_{\omega}}\right)$ took 14 seconds.

Several additional experiments were carried out with different values of the structural parameters. Table 5 summarizes the quality of the multiple model updating results.

\subsection{Model reduction for vibration control}

Vibration control requires a reduced mechanical model of the structure. The reduced stiffness, damping and mass matrices $\left[\mathbf{K}_{r}\right],\left[\mathbf{D}_{r}\right]$ and $\left[\mathbf{M}_{r}\right]$ can be calculated using 
Table 5. The model updating results

\begin{tabular}{ccc}
\hline $\begin{array}{c}\text { Given } \\
\text { perturbation }\end{array}$ & $\begin{array}{c}\text { Result } \\
\text { perturbation }\end{array}$ & $\begin{array}{c}\text { Computation } \\
\text { time }\end{array}$ \\
\hline$\{1.1 E, 0.9 D\}$ & $\{1.1002 E, 0.9061 D\}$ & $0.04 s$ \\
$\{0.7 E, 1.2 D\}$ & $\{0.7012 E, 1.1935 D\}$ & $0.04 s$ \\
$\{0.8 E, 1.3 D\}$ & $\{0.7997 E, 1.2922 D\}$ & $0.04 s$ \\
$\{1.6 E, 0.6 D\}$ & $\{1.5973 E, 0.6114 D\}$ & $0.04 s$ \\
\hline
\end{tabular}

equations

$$
\begin{aligned}
& {\left[\mathbf{K}_{r}\right]=[\boldsymbol{\Phi}]^{T}[\mathbf{K}][\mathbf{\Phi}],} \\
& {\left[\mathbf{D}_{r}\right]=[\boldsymbol{\Phi}]^{T}[\mathbf{D}][\mathbf{\Phi}],} \\
& {\left[\mathbf{M}_{r}\right]=[\boldsymbol{\Phi}]^{T}[\mathbf{M}][\mathbf{\Phi}],}
\end{aligned}
$$

where $[\mathbf{\Phi}]$ is the $n_{\omega} \times n_{\text {dof }}$ matrix of the $n_{\omega}$ eigenmodes of the structure which lie within some frequency range of interest. The FEMPA can be used to calculate these reduced matrices. The coefficients of these matrices lie in $\mathcal{P}\left(n_{d}, n_{p}, \mathbb{R}\right)$ and can be updated rapidly when $\left\{\boldsymbol{\alpha}^{(j)}\right\}$ is known.

\section{Conclusion}

In previous works, the MCRE was used successfully to update models of large industrial structures. In order to do that, however, the objective function had to be approximated very often through mechanical reduction of the model. In this work, we introduced the finite element method over a polynomial algebra in order to calculate an approximate value of the error mathematically. This enables one to update the model of the structure rapidly when the structural parameters change, and also to calculate the reduced mechanical model rapidly. This technique lends itself to multiple application fields which should be explored in the future.

\section{References}

[1] P. Ienny, A.Caro-Bretelle, and E.Pagnacco. Identification from measurements of mechanical fields by finite element model updating strategies. a review. European Journal of Computational Mechanics, 18(3-4):353-376, 2009.

[2] C. Farhat and F. Hemez. Updating finite element dynamics models using an element-by-element sensitivity methodology. AIAA Journal, 31(9):1702-1711, 1993.

[3] D. Claire, F. Hild, and S. Roux. A finite element formulation to identify damage fields : the equilibrium gap method. International Journal for Numerical Methods in Engineering, 61:189208, 2004.

[4] R. J. Allemang. Investigation of Some Multiple Input/Output Frequency Response Function Experimental Modal Analysis Techniques. Phd thesis, University of Cincinnati, 1980.

[5] H. Bui, A. Constantinescu, and H. Maigre. Numerical identification of linear cracks in 2d elastodynamics using the instantaneous reciprocity gap. Inverse problems, 20(4):993-1001, 2004. 
[6] G. Geymonat and S. Pagano. Identification of mechanical properties by displacement field measurement: A variational approach. Meccanica, 38(5):535-545, October 2003.

[7] P. Ladevèze, D. Nedjar, and M. Reynier. Updating of finite element models using vibrations tests. AIAA Journal, 32(7):1485-1491, 1989.

[8] F. Formosa. Contribution à l'amélioration de la modélisation de structures légères en vue de leur contrôle actif. Thèse de doctorat, ENS Cachan, 2004.

[9] A. Deraemaeker. Sur la maitrise des modèles en dynamique des structures à partir de résultats d'essais. Thèse de doctorat, ENS Cachan, 2001.

[10] K. Kim and S. Wang. Second-order variance estimation in poststratified two-stage sampling. Journal of Statistical Planning and Inference, 139(7):Pages 2502-2510, July 2009.

[11] J.C. Helton, J.D. Johnson, C.J. Salaberry, and C.B. Storlie. Survey of sampling based methods for uncertainty and sensitivity analysis. Reliability Engineering and System Safety, 91:1175-1209, 2006.

[12] Z. Xie, R. Wang, X. Yang, and Z. Chien. The perturbation finite element method for solving problems with nonlinear materials. Applied Mathematics and Mechanics, 4:127-140, January 1983.

[13] H. Huang and H. Li. Perturbation finite element method of structural analysis under fuzzy environments. Engineering Applications of Artificial Intelligence, 18(1):83-91, February 2005. 